\title{
FATORES DE RISCO DE QUEDA NA PESSOA COM ACIDENTE VASCULAR CEREBRAL
}

\section{Factores de Riesgo de Caídas en Personas con Accidente Vascular Cerebral}

\section{RISK FACTORS OF FALLING IN THE PERSON WITH CEREBRAL VASCULAR ACCIDENT}

\author{
DOI 10.33194/rper.2020.v3.n1.2.4585 | Submetido 11/09/2019 | Aprovado 28/05/2020
}

\author{
Miguel Ângelo Rodrigues Horta ${ }^{1} \mathbb{D}$; Ana Júlia Nozes ${ }^{2} \mathbb{D}$; Carolina Paulo ${ }^{1} \mathbb{D}$; Maria Elisabete Vilardouro ${ }^{1} \mathbb{D}$; \\ Joana Mendes Marques ${ }^{3}$; ; Luís Manuel Mota de Sousa ${ }^{4}$ (D) \\ 1 - Centro de Medicina e Reabilitação de Alcoitão; 2 - Centro Hospitalar de Lisboa Oeste; \\ 3 - Escola Superior de Saúde da Cruz Vermelha Portuguesa; \\ 4- Escola Superior de Enfermagem S. João de Deus, Comprehensive Health Research Centre, Universidade de Évora
}

\section{RESUMO}

Introdução: 0 acidente vascular cerebral constitui uma das principais causas de incapacidade, provocando alterações que se manifestam numa restrição da funcionalidade da pessoa, contribuindo para um crescimento de quedas acidentais. A identificação de fatores de risco de queda por parte do Enfermeiro Especialista em Enfermagem de reabilitação é imperativa para evitar estes eventos.

Objetivo: Identificar fatores de risco de queda em pessoas com Acidente Vascular Cerebral.

Método: Revisão sistemática da literatura, com pesquisa na plataforma EBSCO Host $\circledast$, na CINAHL e MEDLINE, no período de 1 a 31 de outubro de 2017. Utilizou-se a seguinte equação de pesquisa, (Accidental Falls) AND (Stroke) AND (Risk Factors).

Resultados: Obteve-se um total de 19 estudos e um total de 22 fatores de risco de queda, 12 dos quais presentes na Taxonomia NANDA-I.

Conclusões: Esta pesquisa permitiu identificar fatores de risco de queda na pessoa com AVC. Este estudo constitui um contributo ao desenvolvimento do conhecimento da disciplina de Enfermagem, e que tem implicações na prática clínica, na educação e em novas pesquisas numa área sensível aos cuidados de enfermagem.

Descritores: Acidente Vascular Cerebral; Acidentes por Quedas; Fatores de Risco.

\section{RESUMEN}

Introducción: El accidente cerebrovascular es una de las principales causas de discapacidad, provocando cambios que se manifiestan en una restricción de la funcionalidad de la persona, contribuyendo a un aumento de las caídas accidentales. La identificación de los factores de riesgo de caídas por parte de la enfermera especialista en enfermería de rehabilitación es imprescindible para evitar estos eventos.

Objetivo: Identificar los factores de riesgo de caídas en personas con accidente cerebrovascular.

Método: Revisión sistemática de la literatura, con búsqueda en la plataforma EBSCO Host $\circledast$, CINAHL y MEDLINE, del 1 al 31 de octubre de 2017. La siguiente ecuación de investigación, (Caídas accidentales) Y (Accidente cerebrovascular) Y (Factores de riesgo).

Resultados: se obtuvieron 19 estudios y un total de 22 factores de riesgo de caídas, 12 de los cuales estaban presentes en la taxonomía NANDA-I.

Conclusiones: Esta revisión nos permitió identificar factores de riesgo de caídas en personas con accidente cerebrovascular. Esta contribuye al desarrollo del conocimiento de la disciplina de enfermería y tiene implicaciones para la práctica clínica, la educación y la nueva investigación en un área sensible a la atención de enfermería.

Palabras clave: Accidente Cerebrovascular; Accidentes por Caídas; Factores de Riesgo

\section{ABSTRACT}

Background: Stroke is one of the main causes of disability, causing changes that are manifested in a restriction of the person's functionality, contributing to an increase of accidental falls. The identification of risk factors by the Nurse Specialist in Rehabilitation Nursing is imperative to avoid these events.

Objective: To identify risk factors for falls in people with stroke. 
Methods: Systematic review of the literature, with research on the EBSCO Host ${ }^{\circledR}$ platform, in CINAHL and MEDLINE, in the period of the $1^{\text {st }}$ and $31^{\text {st }}$ of October 2017. The following research equation (Accidental Falls) AND (Stroke) AND (Risk Factors) were used.

Results: A sample of 19 studies and a total of 22 risk factors were obtained, 12 of which are present in the NANDA-I taxonomy.

Conclusions: This research allowed the identification of risk factors for stroke decline. This study contributes to the development of nursing discipline knowledge, and has implications for clinical practice, education and new research in a nursing care sensitive area.

Descriptors: Stroke; Accidental Falls; Risks Factors

\section{INTRODUÇÃO}

A Organização Mundial de Saúde (OMS), refere que o Acidente Vascular Cerebral (AVC) é uma doença clinicamente definida como sendo um comprometimento neurológico focal (ou às vezes global), de ocorrência súbita e duração de mais de 24 horas (ou que causa morte) e com provável origem vascular $^{(1)}$. De acordo com a World Stroke Organization, nos últimos 25 anos, o AVC tornou-se na segunda maior causa de morte e incapacidade no mundo ${ }^{(2)}$.

Cerca de $50 \%$ dos doentes que sobrevivem a um AVC ficam com limitações nas atividades da vida diária, sendo das taxas mais elevadas na União Europeia ${ }^{(3)}$.

Esta doença constitui uma ameaça para a saúde e bemestar da pessoa, não só pela sua elevada incidência, mas também pela elevada taxa de mortalidade e morbilidade $^{(4)}$, a qual pode levar a alterações a nível da funcionalidade, tendo impacto não só nas Atividades de Vida Diária (AVD), como na integração social, podendo desta forma comprometer a qualidade de vida.

As limitações provocadas pelo AVC podem dar origem a outras consequências, não só devido à diminuição da mobilidade, como ao consequente agravamento do estado de dependência e aos riscos associados a estas alterações. Neste seguimento, o risco de queda tornase uma problemática relevante a considerar na pessoa com AVC.

As quedas são a causa subjacente de cerca de 10 a $15 \%$ de todos os episódios que ocorrem nos serviços de

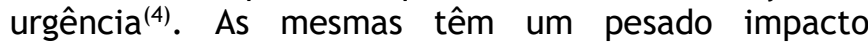
económico nas famílias, na comunidade e na sociedade, além de poderem levar a estados de dependência, perda de autonomia, confusão, imobilização e depressão, que conduzem a restrições variadas nas atividades do dia-a-dia. Em Portugal, de acordo com os dados do sistema nacional de notificação de incidentes, $21 \%$ do total de incidentes notificados são incidentes relacionados com quedas ${ }^{(4)}$.

A Classificação Internacional para a Prática de Enfermagem (CIPE) apresenta o conceito de cair como um foco de enfermagem e define-o como "descida do corpo de um nível superior para um nível inferior devido a desequilíbrio, desmaio ou incapacidade para sustentar pesos e permanecer na vertical", que se traduz "pelo evento ou episódio - Queda"(5).

Por sua vez a NANDA-International (NANDA-I) define o Diagnóstico de Enfermagem, Risco de quedas, como "suscetibilidade aumentada para quedas que pode causar dano físico e comprometer a saúde" (p.799). Os fatores de risco descritos para o mesmo incluem aqueles ligados ao ambiente, ao estado cognitivo e fisiológico dos pacientes, bem como os de causa medicamentosa ${ }^{(6)}$.

Para a prática clínica do Enfermeiro Especialista em Enfermagem de Reabilitação (EEER) à pessoa com AVC, é fundamental uma avaliação do risco de queda, assente na natureza multifatorial deste evento. Sendo desejável a utilização das classificações e linguagens padronizadas, que representem conhecimento atual. Desta forma o enfermeiro com informação relevante para um diagnóstico preciso pode assim intervir de forma adequada, obtendo ganhos positivos na saúde da pessoa com AVC.

A evidência científica é fundamental na sustentabilidade da prática diária do Enfermeiro especialista em Reabilitação(4). Para a revisão sistemática da literatura partimos da seguinte questão norteadora: "Quais os fatores de risco de queda na pessoa com Acidente Vascular Cerebral?”. Esta identificação permite ao EEER a identificação precoce e atuação perante os fatores de risco identificados. 0 enfermeiro, ao cuidar da pessoa com lesão neurológica, desenvolve atividades que promovem a maximização das suas capacidades funcionais, promovendo um melhor desempenho motor, potenciando o rendimento e desenvolvimento pessoal, minimizando desta forma o risco de queda.

O objetivo desta revisão sistemática é identificar os fatores de risco de queda na pessoa com AVC.

\section{MATERIAIS E MÉTODOS}

A investigação científica é efetuada com o objetivo de validar o conhecimento já estabelecido e de produzir novos conhecimentos que, direta ou indiretamente, influenciam a prática(7).

Esta revisão implica em todo o processo a fiabilidade da informação dos artigos consultados. Nesse sentido foi utilizada uma metodologia de pesquisa rigorosa, tendo - cuidado de efetuar a referenciação de forma adequada, assim como, a integridade no tratamento e apresentação dos dados. As Revisões Sistemáticas da Literatura (RSL) permitem identificar, selecionar e avaliar rigorosamente um conjunto de estudos a fim de extrair a melhor evidência científica, para dar resposta a uma questão de investigação. A sua finalidade consiste em reunir toda a evidência empírica mediante a aplicação de métodos sistemáticos e explícitos, com o intuito de minimizar enviesamentos, permitindo obter 
resultados mais fiáveis, tirando assim conclusões mais $\operatorname{adequadas}^{(8)}$.

Procedeu-se a uma revisão sistemática da literatura, por ser um processo criterioso, que permite identificar, avaliar e interpretar todas as pesquisas disponíveis e relevantes, de forma a responder a uma questão que surge num contexto da prática clínica. Os elementos fundamentais de uma revisão sistemática são constituídos por 8 etapas: questão de investigação, definição do problema, objetivos da revisão sistemática; critérios de inclusão e exclusão; estratégia de pesquisa; procedimento de seleção; procedimento de extração dos dados; e procedimento de avaliação da qualidade metodológica dos estudos selecionados ${ }^{(9)}$.

A presente RSL, tem como propósito identificar os fatores de risco de queda em pessoas com AVC. Utilizou-se a seguinte questão de pesquisa: Quais os fatores de risco de queda em pessoas com Acidente Vascular Cerebral?

Para formular a questão de pesquisa foram consideradas as recomendações do Joanna Briggs Institute (JBI) através da estratégia PEO (Population = pessoas com AVC, Exposure = Risco de queda, Outcome $=$ fatores relacionados) .

Além dos critérios definidos pela questão de pesquisa e pela estratégia PEO foram definidos os seguintes critérios de inclusão para a pesquisa de artigos nas bases de dados: estudos no idioma em português e inglês, disponíveis em texto integral, publicados entre janeiro de 2012 e dezembro de 2017 e estudos com maior nível de evidência científica tais como desenho experimental, quase-experimental, de coorte e/ou quantitativos descritivos. Como critério de exclusão, artigos que associavam risco de queda a outras patologias que não o AVC.

A pesquisa eletrónica decorreu no período de 1 a 31 de outubro de 2017, sendo realizada com recurso à plataforma EBSCO Host $^{\circledR}$, nas bases de dados CINAHL Complete e MEDLINE Complete, biblioteca virtual da Escola Superior de Saúde Atlântica, B-On e Biblioteca Virtual da Saúde (BVS).

Para a RSL foram utilizadas palavras-chave e descritores que permitiram uma pesquisa objetiva e facilitaram o processo de seleção dos artigos mais adequados para a questão de investigação. Foi realizada a validação dos descritores na plataforma MeSH (Medical Subject Headings) e DeSC (Descritores em Ciências da Saúde), sendo utilizadas as seguintes palavras-chave e descritores:

AVC/Stroke AND queda acidental/ accidental falls AND risk factors/ fatores de risco

A avaliação dos artigos foi efetuada por dois revisores independentes que compararam as suas análises e chegaram a um consenso sobre a qualidade metodológica dos estudos.

Através dos descritores utilizados foram identificados na plataforma BVS e EBSCO 508 artigos. Após a aplicação dos critérios de inclusão e exclusão foram selecionados 61 artigos. A seleção dos artigos partiu primeiro pela leitura de título, seguido da leitura do resumo. Após esta seleção foram admitidos 24 artigos. Posteriormente, para a extração de dados foi utilizada uma tabela para exclusão de artigos, face aos critérios da JBI, dependendo do tipo de estudo. Estas tabelas foram aplicadas a cada artigo por dois revisores independentes, tendo sido incluídos 19 artigos na revisão sistemática da literatura por apresentar pelo menos 75\% dos critérios. Atendeu-se ao protocolo PRISMA para o processo de identificação, seleção, elegibilidade e inclusão dos estudos. (Figura 1)

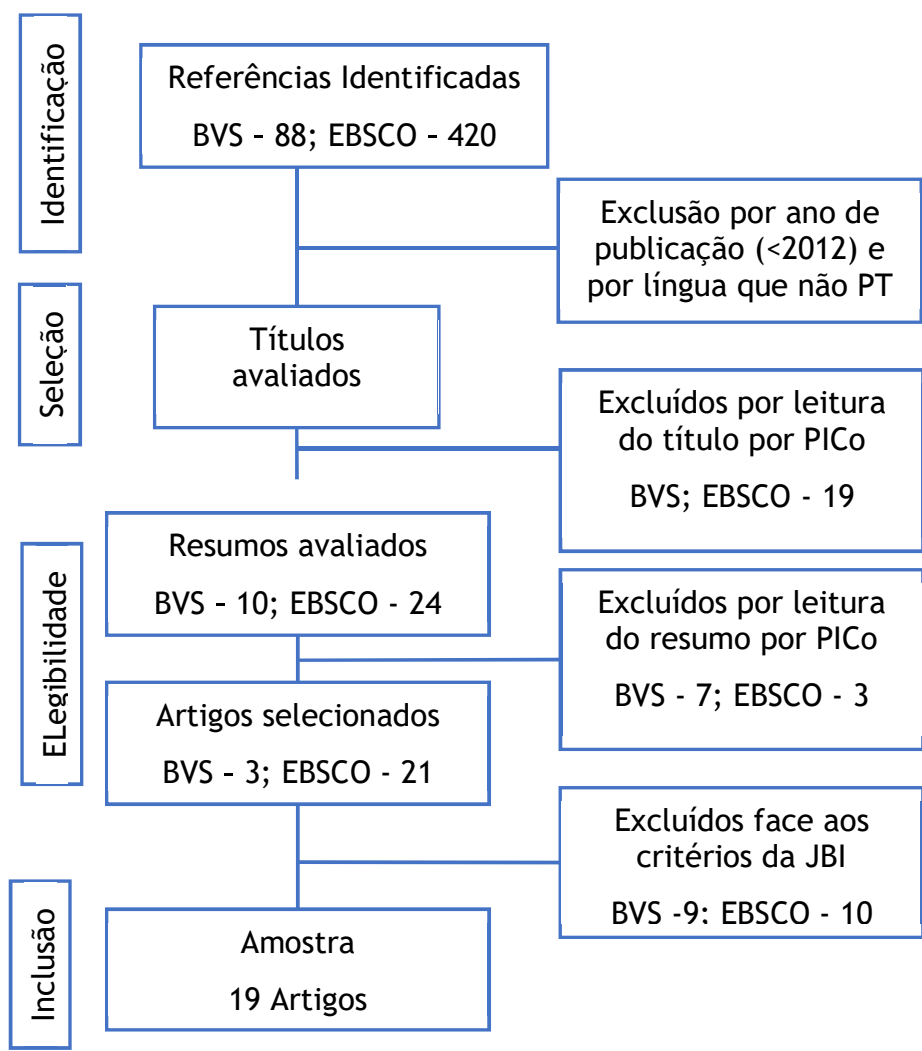

Fonte: Dados de pesquisa, 2017

Figura 1- Mapeamento da identificação, análise e seleção de artigos

Visto o objeto de estudo serem os artigos, considerouse o princípio do respeito pela propriedade intelectual dos autores dos artigos que constituem a amostra, através da citação completa e rigorosa dos mesmos.

\section{RESULTADOS}

Dos 19 artigos incluídos nesta revisão, 7 foram

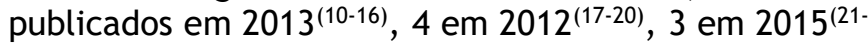
23), 2 em 2016(24-25), 2 em 2017(26-27) e um último em $2014^{(28)}$.

Relativamente ao país encontraram-se 10 origens, dos quais se destacam os Estados Unidos da América com 8 estudos, (11-12,14-16, 19,25-26), Singapura ${ }^{(10,22)}$ e $\operatorname{Brasil}^{(20,28)}$ com 2 estudos cada. Seguem-se Irlanda(21), Bélgica(13), Turquia(17), Coreia do Sul(18), Suécia( $^{(23)}$ e Tailândia(27) com 1 artigo cada.

Os artigos incluídos para análise de dados nesta RSL são estudo primários que utilizam metodologia quantitativa, 8 estudos de coorte ${ }^{(13,15-17,21,26-28)}$ e 11 estudos descritivos $(10-12,14,18-20,22-25)$. 
As amostras dos estudos selecionados variaram de $14^{(25)}$ a $16782^{(12)}$ participantes sobreviventes de AVC.

Nesta RSL apenas um estudo foi realizado no âmbito da enfermagem e teve como objetivo identificar o diagnóstico de enfermagem "Risco de Queda em idosos com AVC"(20).

Identificaram-se 22 fatores de risco de queda associados ao AVC nesta revisão, os quais foram organizados de acordo com as categorias da NANDA-I (Quadro 1).

\section{Fatores de Risco}

N NANDA-I

\section{Ambientais}

\begin{tabular}{|c|c|c|}
\hline Ambiente social agitado(26) & 1 & \\
\hline Ambiente cheio de objetos ${ }^{(26)}$ & 1 & 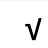 \\
\hline
\end{tabular}

\section{Fisiológicos}

Dificuldades na

$\operatorname{marcha}^{(10,11,14,20,24,25,27)}$

Redução da força em extremidade inferior $^{(10,20,25)}$

Mobilidade reduzida(11,16,21,28)

$7 \quad$ v

Populações de risco

Idade $\geq 65$ anos $^{(14,20,21,22,24)}$

$3 \mathrm{v}$

ondições associadas

Agentes farmacológicos_Antihipertensores) ${ }^{(26-27)}$

Alteração da função

cognitiva/sensorial $(10,16,20)$

Anemia $^{(12)}$

Deficit propriocetivo(20)

Equilíbrio prejudicado(10-11,16,1920,22,26-27)

Uso de dispositivo auxiliar (ex. andarilho, canadianas, cadeira de rodas) $)^{(13)}$

\begin{tabular}{|l|l|l} 
Visão prejudicada(20) $^{(20}$ & 1 & $\mathbb{V}$ \\
\hline Aumento tónus $^{(27)}$ & 1 & \\
\hline Dificuldade nas transferências $^{(10-11)}$ & 2 & \\
\hline $\begin{array}{l}\text { Declínio das atividades básicas de } \\
\text { vida diária(10,15,22,27) }^{(10,2)}\end{array}$ & 4 & \\
\hline
\end{tabular}

\begin{tabular}{|c|c|}
\hline Internamento prolongado(10-11) & 2 \\
\hline Lesão do hemisfério direito $(15,18,28)$ & 3 \\
\hline Lesão hemisfério esquerdo ${ }^{(17)}$ & 1 \\
\hline Maior tempo pós-AVC(28) & 1 \\
\hline \multicolumn{2}{|l|}{ sicológicos } \\
\hline Medo de cair $(16,18)$ & 2 \\
\hline Depressão ${ }^{(26,27)}$ & 2 \\
\hline
\end{tabular}

Fonte: Dados de pesquisa, 2017

Quadro 1 - Síntese comparativa dos fatores de risco encontrados na RSL e os que estão classificados na NANDA-I. Lisboa, 2018.
No que respeita à organização dos fatores de risco de queda, a NANDA-I organiza os indicadores em seis categorias. Após a pesquisa e análise dos artigos selecionados foi possível acrescentar mais uma categoria, que foi denominada por fatores psicológicos.

$\mathrm{Na}$ categoria de fatores ambientais são nos apresentados 5 indicadores de risco de queda. A presença de um ambiente cheio de objetos $(n=1)$ foi o único encontrado. Contudo no mesmo artigo foi identificado um fator adicional, que um ambiente social calmo $(n=1)$ leva a que a pessoa com AVC se sinta mais segura e saia de casa estando assim sujeita aos perigos ambientais que propiciam as quedas.

A categoria de fatores fisiológicos apresenta 10 fatores de risco na NANDA-I. Destes foram encontrados os seguintes, dificuldades na marcha $(n=7)$, mobilidade reduzida $(n=4)$ e redução da força em extremidade inferior $(n=3)$.

$\mathrm{Na}$ categoria que engloba a população de risco são apresentados 5 fatores de risco, tendo sido identificado o fator idade superior ou igual a 65 anos $(n=5)$.

No grupo das condições associadas, a taxonomia apresenta 17 fatores de risco de queda, dos quais foram identificados sete: equilíbrio prejudicado $(n=8)$, alteração da função cognitiva $(n=3)$, ação de agentes farmacológicos (anti hipertensores) $(n=2)$, anemia $(n=1)$, deficit propriocetivo $(n=1)$, visão prejudicada $(n=1)$ e uso de dispositivo auxiliar $(n=1)$. Foram identificados mais sete fatores de risco de queda não presentes na taxonomia NANDA-I: declínio das atividades básicas de vida diária $(n=4)$ e lesão do hemisfério direito $(n=3)$, dificuldade nas transferências $(n=2)$, internamentos prolongados $(n=2)$, aumento do tónus $(n=1)$, lesão do hemisfério esquerdo $(n=1)$ e maior tempo pós AVC $(n=1)$.

$\mathrm{Na}$ categoria criada referente aos fatores psicológicos, os estudos apontam para o medo de cair $(n=2)$ e depressão $(n=2)$ como fatores de risco de queda para a pessoa com AVC.

\section{DISCUSSÃO}

A discussão dos resultados deve incidir nas implicações da evidência apresentada em relação às decisões para a prática clínica, assim, procurando responder à questão de partida verificamos que os fatores de risco de queda na pessoa com AVC são múltiplos, sendo que os mais identificados são: as dificuldades na marcha, equilíbrio prejudicado, idade $\geq 65$ anos, declínio das atividades básicas de vida diária, mobilidade reduzida, lesões do hemisfério direito, alterações cognitivas e redução da força em extremidades inferiores.

As alterações de equilíbrio decorrentes do AVC evidenciam-se como o maior fator de risco, uma vez que a pessoa tem dificuldade em controlar e repor o equilíbrio no decorrer da marcha através de alterações repentinas de postura e centro de massa ${ }^{(10-11,16,19-20,22,26-}$ 27), a manutenção do equilíbrio e do controlo postural são necessárias para o desempenho adequado das atividades de vida diária(20), daí se pode afirmar que um número significativo de quedas resulta de dificuldades 
em corrigir alterações posturais na realização de atividades básicas de vida diária.

Pela análise dos artigos verificou-se que alterações a nível das características da marcha tais como passada $^{(11)}$, velocidade ${ }^{(14)}$, variação/estabilidade ${ }^{(24)}$, simetria $(24,27)$ ou mesmo alterações na marcha no $\operatorname{geral}^{(10,20,25)}$ são, indicadores relevantes de risco de queda na pessoa com AVC. Outros fatores como aumento do tónus também podem afetar esta função ${ }^{(27)}$.

Apesar das dificuldades de marcha e equilíbrio prejudicado serem indicadores de risco de queda em pessoas com AVC, foi verificado que 6 anos após a doença, indivíduos que apresentavam poucas alterações a nível destes fatores, apresentaram maior número de quedas que indivíduos que apresentavam maior compromisso da marcha e do equilíbrio. Tal deveu-se ao fato de o grupo que apresentou melhor avaliação de marcha e equilíbrio não estar tão desperto para o perigo de queda e o grupo que apresentou melhores resultados desenvolveu medidas para compensar as alterações ${ }^{(23)}$. As descobertas sugerem que a monitorização do risco de queda após o AVC é importante, mesmo quando uma avaliação inicial da marcha e do equilíbrio pós AVC é satisfatória(23). Por outro lado, refere que quanto mais tempo passa desde o início da doença, maior é o risco de queda na pessoa com AVC (28).

O AVC resulta numa diminuição de controlo das extremidades, que limita a capacidade funcional da pessoa e dificulta a realização das atividades de vida diária, o que associado a outros fatores aumenta o risco de queda ${ }^{(10,20,25)}$.

Quanto à localização de lesão, estudos demonstram que as lesões do hemisfério direito são mais indicadoras de risco de queda ${ }^{(15,18,28)}$. Esta conclusão está associada ao fato de pessoas com AVC do hemisfério direito apresentarem comportamentos mais impulsivos e maiores défices visuo-espaciais que aumentem o risco de queda ${ }^{(15)}$, apresentam uma heminegligência espacial que se traduz num défice de atenção e consciência para o lado parético ${ }^{(18)}$. Estes défices vão contribuir para uma instabilidade da marcha, potenciando assim o risco de queda(28). Estas pessoas tendem a ser impulsivas, desorganizadas, sobrevalorizando as suas capacidades e não aprendem com os erros e com instrução, fatores que os impedem de ser tornar independentes nas AVD's, dado o risco elevado de queda(17).

Outro artigo encontrado refere que pessoas com AVC do hemisfério esquerdo caíam mais (47\%) face aos do hemisfério direito $(21 \%)^{(17)}$. Neste artigo pessoas com lesão do hemisfério esquerdo apresentaram um risco de queda quatro vezes superior num período de 6 meses. Os autores associam um maior número de quedas em pessoas com AVC do hemisfério esquerdo por estes apresentarem problemas de comunicação, mas por outro lado apresentam perceção visual e memória intactas. Estas pessoas aprendem assim por demonstração e erro, sendo capazes de sintetizar partes das tarefas, o que lhes permite tornarem-se mais independentes, requerendo assim uma menor supervisão por parte dos profissionais. Este fato permite explicar assim uma maior incidência de quedas em pessoas com AVC do hemisfério esquerdo versus hemisfério direito ${ }^{(17)}$.

$O$ défice cognitivo, resultante do AVC, é também considerado um fator de risco de queda. Indivíduos com alterações cognitivas têm uma perceção reduzida das suas incapacidades, levando-os a desempenhar tarefas para além das suas capacidades ${ }^{(10)}$, podem provocar défices de atenção que podem dar origem a quedas acidentais no decorrer de $\operatorname{AVD}^{(16)}$, compromete a marcha, equilíbrio e estabilidade postural ${ }^{(20)}$. Observou-se que o défice cognitivo é um forte indicador de risco de queda.

A pessoa com AVC apresenta dificuldade para executar atividades pois pode apresentar uma desorientação espacial, estando a sua capacidade de resposta protetora comprometida que associado a uma alteração da marcha, equilíbrio e estabilidade postural leva à ocorrência de quedas.

A análise dos artigos indica uma alta sensibilidade para instrumentos de avaliação, permitindo identificar fatores de risco de queda, tais como, BBS (Berg Balance Scale) ${ }^{(10,22)}$, TUG (Timed Up \& Go Test $)^{(16,28)}$ e MIF (Medida Independência Funcional) $)^{(10,15,22,27)}$. O instrumento de avaliação de TUG avalia a capacidade de equilíbrio estático e dinâmico da pessoa, envolvendo manobras funcionais como levantar-se, andar, dar a volta e sentar-se. A escala de Berg é um instrumento de avaliação do equilíbrio estático e dinâmico. Os resultados encontrados revelam que fracos desempenhos nestes testes refletem-se em risco de queda, uma vez que o equilíbrio deficiente está associado a quedas ${ }^{(22)}$. Relativamente à MIF, na presente revisão verificou-se que baixos valores de pontuação global ${ }^{(22,27)}$ são indicadores de risco de queda. Dentro das diferentes categorias que constituem o instrumento de avaliação, verifica-se que existe maior risco de queda para pessoas com baixas pontuações a nível do controlo vesical e intestinal, transferências, mobilidade, higiene e vestuário( ${ }^{(10)}$, bem como o uso do sanitário, transferências para a cama, banheira/duche e uso de escadas ${ }^{(15)}$. Sendo assim é possível afirmar que estes instrumentos de avaliação são pertinentes para determinar o risco de queda na população em estudo.

A nível do ambiente físico verificou-se que as quedas ocorrem com mais frequência no domicílio. Pela análise dos artigos os locais mais comuns onde ocorrem quedas são na casa de banho ${ }^{(10,16,18)}$ e no quarto ${ }^{(11)}$, o que vem reforçar o já mencionado anteriormente, que baixos valores de MIF no uso de sanitário e transferências para a cama, banheira/duche ${ }^{(15)}$, são indicadores de risco de queda. Apesar destes estudos apontarem estas divisões como locais de queda frequente em pessoas com AVC, não referem se estas se devem a falta de material antiderrapante, falta de barras de apoio na sanita ou banheiras/polibans, presença de tapetes, divisões com muito mobiliário que dificultam a circulação pela divisão. Estes dados seriam fundamentais para a RSL, uma vez que é importante uma avaliação do domicílio pelos profissionais, educação sobre segurança, bem como modificação do ambiente doméstico durante o planeamento de alta, contribuindo para que não corram 
quedas em casa(10). Foi também verificado que em locais com ambientes sociais menos seguros existe um menor número de quedas, tal deve-se ao fato da pessoa com AVC não se sentir tão segura em ir para a rua não correndo assim o risco em cair durante a marcha na rua $^{(26)}$.

As incapacidades, de nível motor e sensitivo e visual, características do AVC, levam a pessoa a estar mais vulnerável e a apresentar episódios de queda, que podem resultar em hospitalização, demora no processo de reabilitação, diminuição da participação social ou morte.

$\mathrm{Na}$ RSL encontrada sobre esta temática( ${ }^{(30)}$ os autores encontraram vários fatores de risco para quedas em pessoas com AVC. O equilíbrio e a maior dependência nas atividades de vida diárias são apresentados como os mais frequentes, resultado que vem reforçar os achados obtidos no nosso estudo. Esta RSL aponta outros fatores de risco encontrados na nossa pesquisa, nomeadamente, a dificuldade na marcha, mobilidade reduzida, a diminuição de força nas extremidades, as alterações cognitivas e sensoriais, medo de cair, a depressão e a heminegligência como fator de risco, fator que na presente pesquisa se associou às lesões do hemisfério direito e alterações cognitivas. Ao contrário da presente pesquisa, o estudo refere que não há associação entre o local da lesão do AVC e da idade como risco de queda.

Desta forma, face à literatura encontrada, considerada de qualidade e atual, foi possível identificar algumas semelhanças que reforçam os achados e solidificam os resultados obtidos.

Verifica-se que existem fatores de risco que não estão classificados na NANDA-I, tais como fatores ambientais físicos/sociais ${ }^{(26)}$, lesões do hemisfério direito ${ }^{(15,18,28)}$, declínio das atividades básicas de vida diária(10,15,22,27), internamentos prolongados ${ }^{(10,11)}$, dificuldade nas transferências $^{(10,11)}$, medo de cair $^{(16,18)}$, depressão(26,27), aumento do tónus ${ }^{(27)}$, AVC do hemisfério esquerdo ${ }^{(17)}$ e maior tempo pós AVC ${ }^{(28)}$

Com base nos resultados da RSL e da proposta NANDA-I sobre os fatores de risco de queda da pessoa com AVC, confirma-se a existência multifatorial dos fatores de risco, o que requer intervenções abrangentes.

Desta forma os resultados contribuem para o conhecimento dos enfermeiros, na medida em que os achados podem ser aplicados na prática clínica, permitindo desta forma uma prática baseada na evidência.

Ao Enfermeiro Especialista em Enfermagem de Reabilitação são atribuídas competências específicas, pois "os cuidados de enfermagem de reabilitação constituem uma área de intervenção especializada que decorre de um corpo de conhecimentos $e$ procedimentos específicos. Tem por foco de atenção a manutenção e promoção do bem-estar e da qualidade de vida, a recuperação da funcionalidade, tanto quanto possível através da promoção do autocuidado, da prevenção de complicações e da maximização das capacidades." (31). Assim sendo, este profissional deve conhecer os fatores de risco de queda no AVC identificados neste estudo e realizar medidas de prevenção para os mesmos, contudo não limitar o pensamento, a avaliação e a ação com base neles. Esta intervenção especializada permite assim melhorar a funcionalidade da pessoa com AVC através de ações específicas do enfermeiro especialista de reabilitação, maximizando a funcionalidade da pessoa e minimizando fatores de risco.

0 fator de risco ambiente pode ser minimizado pelo EEER, caso este assuma a sua função de promover um ambiente seguro, por meio de estratégias de visita ao domicílio e de educação em saúde. Na maioria das vezes a pessoa com AVC não cai por realizar atividades perigosas, mas sim no executar de atividades básicas de vida diária. Identificar os fatores de risco ambientais bem como capacitar a pessoa a desenvolver as atividades em segurança através de técnicas seguras, possibilita uma intervenção por parte deste perito no sentido de eliminar alguns destes fatores e assim evitar eventos de queda.

A utilização de instrumentos de avaliação adequados por parte do EEER por sua vez, facilita a avaliação da funcionalidade da pessoa com AVC, permitindo também identificar a existência de risco de queda.

\section{CONCLUSÃO}

A RSL contribuiu para a sistematização do conhecimento e comparação de vários estudos, permitindo desta forma a organização do estado da arte numa determinada problemática.

Os artigos analisados são estudos realizados com instrumentos em contextos diferentes, com dados colhidos em diversos países do mundo, permitindo desta forma um vasto conhecimento da problemática, sendo esta a razão encontrada para a existência de um elevado número de fatores.

Foram encontrados inúmeros estudos acerca de fatores de risco de queda na pessoa com AVC. O fato da maior parte dos artigos encontrados não serem do âmbito de Enfermagem constitui uma limitação ao estudo.

Com base nos resultados da RSL e dando resposta à questão de investigação, foram identificados os seguintes fatores de risco de queda em pessoas com AVC: dificuldades na marcha, equilíbrio prejudicado, idade superior a 65 anos, mobilidade reduzida, declínio das atividades básicas de vida diária, redução da força em extremidade inferior, lesões no hemisfério direito e alterações cognitivas. Confirma-se assim a natureza multifatorial dos fatores de risco, que requer intervenções abrangentes e multidisciplinares. Desta forma, numa pessoa com AVC torna-se necessária uma abordagem multiprofissional e uma avaliação do seu estado geral, dos conhecimentos, capacidades e défices.

Durante o período de pesquisa, encontrou-se apenas um artigo no âmbito de Enfermagem. Assim sendo e dado o número de fatores de risco encontrados, sugere-se que sejam desenvolvidos mais estudos nesta área. 


\section{REFERÊNCIAS BIBLIOGRÁFICAS}

1. World Health Organization. WHO STEPS Stroke Manual: The WHO STEP wise approach to stroke surveillance. Geneva, World Health Organization, 11; 2006.

2. World Stroke Organization. Oral statement of the World Stroke Organisation (WSO) and the European Stroke Organisation (ESO). Budapeste, World Stroke Organization; 2017 [consultado a 20 de março de 2019]. Disponível em: https://www.worldstroke.org/images/WSO_ESO_OralStatement_WHO-

EURO_Regional_Committee_2017_final.pdf.

3. Luís JM. Relação entre a quantidade e qualidade do uso do membro superior parético após AVC e a capacidade de resiliência. Mestrado em Enfermagem de Reabilitação, Escola Superior de Saúde de Bragança, Bragança, Portugal; 2015 [consultado a 27 de novembro de 2017]. Disponível em:

https://bibliotecadigital.ipb.pt/bitstream/10198/12044/1/Joana\%2 OMargarida\%20Lopes\%20Lu\%C3\%ADs.pdf.

4. Ministério da Saúde (PT). Despacho $n^{\circ}$ 1400-A/2015. Plano Nacional para a Segurança dos Doentes (PNSD) 2015-2020. Diário da República, $2^{\mathrm{a}}$ série. 2015 Fevereiro 10 (28):3882.

5. Conselho Internacional de Enfermeiros. Classificação Internacional para a Prática de Enfermagem Versão 2015. Lisboa: Ordem dos Enfermeiros. 2016.

6. Herdman TH, Kamitsuru S. Diagnósticos de enfermagem da NANDAI: definições e classificação 2018-2020. Porto Alegre: Artmed. 2018. $799 \mathrm{p}$.

7. Fortin MF. 0 processo de investigação: Da Concepção á Realização (5 Edição). Loures: Lusociência - Edições técnicas e científica Lda; 2009.

8. Sousa LM, Firmino CF, Marques-Vieira CM, Severino SS, Pestana HC. Revisões da literatura científica: tipos, métodos e aplicações em enfermagem. Rev Port Enferm Reabil. 2018 Jun 22;1(1):45-54. DOI: 10.33194/rper.2018.v1.n1.07.4391

9. Green S, Higgins JPT, Alderson P, Clarke M, Mulrow CD, Oxman AD. Cochrane Handbook for Systematic Reviews of Interventions Version 5.0.1 updated March 2011[Internet]. Melbourne: TheCochraneCollaboration;2011[consultado a 27 de novembro de 2017]. Disponivel em: http://handbook.cochrane.org/

10. Chin LF, Wang JY, Ong CH, Lee WK, Kong KH. Factors affecting falls in community-dwelling individuals with stroke in Singapore after hospital discharge. Singapore Med J. 2013 Oct 1;54(10):569-75.

11. Mansfield A, Inness EL, Wong JS, Fraser JE, Mcllroy WE. Is impaired control of reactive stepping related to falls during inpatient stroke rehabilitation?. Neurorehabil Neural Repair. 2013 Jul;27(6):526-33. doi: 10.1177/1545968313478486.

12. Bowling $C B$, Brown $C J$, Allman RM, Warriner $A H$, Curtis $J R$, Warnock DG, Muntner $P$, Bradbury BD, Kilpatrick RD, Isitt JJ, Judd S. Low hemoglobin levels and recurrent falls in US men and women: prospective findings from the REasons for geographic and racial differences in stroke (REGARDS) cohort. Am J Med Sci. 2013 Jun 1;345(6):446-54. doi: 10.1097/MAJ.0b013e3182638364

13. Baetens T, De Kegel A, Palmans T, Oostra K, Vanderstraeten G, Cambier D. Gait analysis with cognitive-motor dual tasks to distinguish fallers from nonfallers among rehabilitating stroke patients. Arch Phys Med Rehabil. 2013 Apr 1;94(4):680-6.. doi: 10.1016/j.apmr.2012.11.023

14. Said CM, Galea MP, Lythgo N. People with stroke who fail an obstacle crossing task have a higher incidence of falls and utilize different gait patterns compared with people who pass the task. Phys Ther. 2013 Mar 1;93(3):334-44.. doi: 10.2522/ptj.20120200

15. Rosario ER, Kaplan SE, Khonsari S, Patterson D. Predicting and assessing fall risk in an acute inpatient rehabilitation facility. Rehabil Nurs. 2014 Mar;39(2):86-93. doi: 10.1002/rnj.114.

16. Jalayondeja $C$, Sullivan $P E$, Pichaiyongwongdee S. Six-month prospective study of fall risk factors identification in patients poststroke. Geriatr Gerontol Int. 2014 Oct;14(4):778-85.. doi: 10.1111/ggi.12164.

17. Alemdaroğlu E, Uçan H, Topçuoğlu AM, Sivas F. In-hospital predictors of falls in community-dwelling individuals after stroke in the first 6 months after a baseline evaluation: a prospective cohort study. Arch Phys Med Rehabil. 2012 Dec 1;93(12):2244-50. doi: 10.1016/j.apmr.2012.06.014.

18. Lim JY, Jung SH, Kim WS, Paik NJ. Incidence and risk factors of poststroke falls after discharge from inpatient rehabilitation. PM\&R. 2012 Dec;4(12):945-53. doi: 10.1016/j.pmrj.2012.07.005.
19. Mansfield A, Mochizuki G, Inness EL, Mcllroy WE. Clinical correlates of between-limb synchronization of standing balance control and falls during inpatient stroke rehabilitation. Neurorehabil Neural Repair. 2012 Jul;26(6):627-35. doi: $10.1177 / 1545968311429688$.

20. Morais HC, Holanda GF, de Souza Oliveira AR, de Sousa Costa AG, Ximenes CM, de Araujo TL. Identificação do diagnóstico de enfermagem "risco de quedas em idosos com acidente vascular cerebral". Rev Gaúcha Enferm. 2012 Jun;33(2):117-24.. doi: $10.1590 /$ S1983-14472012000200017.

21. Callaly EL, Ni Chroinin D, Hannon N, Sheehan O, Marnane M, Merwick A, et al. Falls and fractures 2 years after acute stroke: the North Dublin Population Stroke Study. Age ageing. 2015 Aug 12;44(5):882-6. doi: 10.1093/ageing/afv093.

22. Maeda N, Urabe Y, Murakami M, Itotani K, Kato J. Discriminant analysis for predictor of falls in stroke patients by using the Berg Balance Scale. Singapore Med J. 2015 May;56(5):280-3. doi: 10.11622/smedj.2015033.

23. Minet LR, Peterson E, von Koch L, Ytterberg C. Occurrence and predictors of falls in people with stroke: six-year prospective study. Stroke. 2015 Sep;46(9):2688-90. doi: 10.1161/STROKEAHA.115.010496.

24. Punt M, Bruijn SM, van Schooten KS, Pijnappels M, van de Port IG, Wittink H, van Dieën JH. Characteristics of daily life gait in fall and non fall-prone stroke survivors and controls. J Neuroeng Rehabil. 2016 Dec;13(1):67. doi: 10.1186/s12984-016-0176-z.

25. Salot $P$, Patel $P$, Bhatt $T$. Reactive balance in individuals with chronic stroke: biomechanical factors related to perturbationinduced backward falling. Phys Ther. 2016 Mar 1;96(3):338-47. doi: 10.2522/ptj.20150197.

26. Wing JJ, Burke JF, Clarke PJ, Feng C, Skolarus LE. The role of the environment in falls among stroke survivors. Arch Gerontol Geriatr. 2017 Sep 1;72: 1-5. doi: 10.1016/j.archger.2017.04.007.

27. Wei TS, Liu PT, Chang LW, Liu SY. Gait asymmetry, ankle spasticity, and depression as independent predictors of falls in ambulatory stroke patients. PloS one. 2017 May 23;12(5): e0177136. doi: $10.1371 /$ journal.pone.0177136.

28. Beatriz Pinto E, Nascimento C, Marinho C, Oliveira I, Monteiro M, Castro M, Myllane-Fernandes P, Ventura LM, Maso I, Alberto Lopes A, Oliveira-Filho J. Risk factors associated with falls in adult patients after stroke living in the community: baseline data from a stroke cohort in Brazil. Top Stroke Rehabil. 2014 May 1;21(3):220-7. doi: $10.1310 /$ tsr2103-220.

29. Registered Nurses' Association of Ontario. Falls Prevention. Building the Foundations for Patient Safety. Self-Learning Package. Ontario: Registered Nurses' Association of Ontario;2007.

30. Batchelor FA, Mackintosh SF, Said CM, Hill KD. Falls after stroke. International Journal of Stroke. 2012 Aug;7(6):482-90. Disponível em: https://doi.org/10.1111/j.1747-4949.2012.00796.x

31. Ordem dos Enfermeiros Regulamento das competências específicas do enfermeiro especialista em enfermagem de reabilitação. Lisboa: Ordem dos Enfermeiros. 2010 Dec [consultado a 12 de novembro 2017 às 15 horas]:1-4. disponível em: http://www.ordemenfermeiros.pt/colegios/Paginas/MCEEdeR_Legis lacao.aspx 